\title{
Some inequalities for the ratio of gamma functions
}

Xiaodong Cao* and Ruopeng Wang

\section{"Correspondence:}

caoxiaodong@bipt.edu.cn

Department of Mathematics and

Physics, Beijing Institute of

Petro-Chemical Technology, Beijing, 102617, P.R. China

\begin{abstract}
In this paper, we present some sharp inequalities for the ratio of gamma functions. The main tool is the multiple-correction method formulated in (Cao et al. in J. Number Theory 149:327-350, 2015; Cao in J. Math. Anal. Appl. 424:1425-1446, 2015). Some conjectures are proposed.
\end{abstract}

MSC: 33B15; $26 \mathrm{D} 07$

Keywords: gamma function; Wallis ratio; inequalities; continued fraction; rate of convergence

\section{Introduction}

The problem of finding new and sharp inequalities for the ratio of gamma functions and, in particular, for the Wallis ratio

$$
W(n):=\frac{(2 n-1) ! !}{(2 n) ! !}=\frac{\Gamma\left(n+\frac{1}{2}\right)}{\sqrt{\pi} \Gamma(n+1)}, \quad n \in \mathbb{N}=\{1,2, \ldots\}
$$

has attracted the attention of many researchers (see, e.g., [1-13] and the references therein). Here, $\Gamma$ is the gamma function, and we employ the special double factorial function as follows:

$$
\begin{aligned}
& (2 n) ! !=2 \cdot 4 \cdot 6 \cdots(2 n)=2^{n} \Gamma(n+1), \\
& (2 n-1) ! !=1 \cdot 3 \cdot 5 \cdots(2 n-1)=\pi^{-1 / 2} 2^{n} \Gamma\left(n+\frac{1}{2}\right) .
\end{aligned}
$$

See [14], p.258.

Chen and Qi [15] established the following inequalities:

$$
\frac{1}{\sqrt{\pi\left(n+\frac{4}{\pi}-1\right)}} \leq W(n)<\frac{1}{\sqrt{\pi\left(n+\frac{1}{4}\right)}}, \quad n \in \mathbb{N},
$$

where $\frac{4}{\pi}-1$ and $\frac{1}{4}$ are best possible.

(c) 2015 Cao and Wang. This article is distributed under the terms of the Creative Commons Attribution 4.0 International License (http://creativecommons.org/licenses/by/4.0/), which permits unrestricted use, distribution, and reproduction in any medium, provided you give appropriate credit to the original author(s) and the source, provide a link to the Creative Commons license, and indicate if changes were made. 
For $n \in \mathbb{N}$, let

$$
\begin{aligned}
& T_{1}(n):=\frac{1 \cdot 4 \cdots(3 n-2)}{3 \cdot 6 \cdots(3 n)}=\frac{\Gamma\left(n+\frac{1}{3}\right)}{\Gamma(n+1) \Gamma\left(\frac{1}{3}\right)}, \\
& T_{2}(n):=\frac{2 \cdot 5 \cdots(3 n-1)}{3 \cdot 6 \cdots(3 n)}=\frac{\Gamma\left(n+\frac{2}{3}\right)}{\Gamma(n+1) \Gamma\left(\frac{2}{3}\right)} .
\end{aligned}
$$

Very recently, Mortici et al. [7] proved that for every integer $n \geq 1$,

$$
\frac{\beta}{\sqrt[3]{n^{2}+\frac{1}{3} n}} \exp \left(-\frac{2}{81 n^{2}}\right)<T_{1}(n)<\frac{\beta}{\sqrt[3]{n^{2}+\frac{1}{3} n}} \exp \left(-\frac{2}{81 n^{2}}+\frac{2}{243 n^{3}}\right)
$$

and

$$
\frac{\tau}{\sqrt[3]{n+\frac{1}{3}}} \exp \left(-\frac{1}{81 n^{2}}\right)<T_{2}(n)<\frac{\tau}{\sqrt[3]{n+\frac{1}{3}}} \exp \left(-\frac{1}{81 n^{2}}+\frac{2}{243 n^{3}}\right)
$$

where

$$
\beta=\frac{1}{\Gamma\left(\frac{1}{3}\right)}=\frac{\sqrt{3}}{2 \pi} \Gamma\left(\frac{2}{3}\right)=0.37328 \ldots \quad \text { and } \quad \tau=\frac{1}{\Gamma\left(\frac{2}{3}\right)}=0.73848 \ldots
$$

From (1.5) and (1.6), we have the following asymptotic formulas:

$$
\begin{aligned}
& T_{1}(n)=\frac{\beta}{\sqrt[3]{n^{2}+\frac{1}{3} n}} \exp \left(-\frac{2}{81 n^{2}}+\frac{2}{243 n^{3}}\right)\left(1+O\left(\frac{1}{n^{4}}\right)\right), \\
& T_{2}(n)=\frac{\tau}{\sqrt[3]{n+\frac{1}{3}}} \exp \left(-\frac{1}{81 n^{2}}+\frac{2}{243 n^{3}}\right)\left(1+O\left(\frac{1}{n^{4}}\right)\right) .
\end{aligned}
$$

Motivated by these works, the purpose of this paper is to present some sharp inequalities for $W(n), T_{1}(n)$ and $T_{2}(n)$ by using the multiple-correction method developed in $[16,17]$.

Throughout the paper, the notation $\lfloor x\rfloor$ denotes the largest integer not exceeding $x$. The notation $P_{k}(x)$ (or $Q_{k}(x)$ ) denotes a polynomial of degree $k$ in $x$, while the notation $\Psi(k ; x)$ means a polynomial of degree $k$ in $x$ with all coefficients non-negative, which may be different at each occurrence. Let $\left(a_{n}\right)_{n \geq 1}$ and $\left(b_{n}\right)_{n \geq 0}$ be two sequences of real numbers with $a_{n} \neq 0$ for all $n \in \mathbb{N}$. The generalized continued fraction

$$
\tau=b_{0}+\frac{a_{1}}{b_{1}+\frac{a_{2}}{b_{2}+}}=b_{0}+\frac{a_{1}}{b_{1}+} \frac{a_{2}}{b_{2}+}=\cdots=b_{0}+\underset{n=1}{\mathbf{K}}\left(\frac{a_{n}}{b_{n}}\right)
$$

is defined as the limit of the $n$th approximant

$$
\frac{A_{n}}{B_{n}}=b_{0}+\mathbf{K}_{k=1}^{n}\left(\frac{a_{k}}{b_{k}}\right)
$$


as $n$ tends to infinity. The numerators $A_{n}$ and denominators $B_{n}$ of the approximants satisfy the recurrence relations

$$
A_{n+2}=b_{n+2} A_{n+1}+a_{n+2} A_{n}, \quad B_{n+2}=b_{n+2} B_{n+1}+a_{n+2} B_{n}
$$

with initial values $A_{0}=b_{0}, B_{0}=1, A_{1}=b_{0} b_{1}+a_{1}$ and $B_{1}=b_{1}$. See [18], p.105.

\section{Inequalities for $W(n)$}

In this section, in order to illustrate the idea of this paper, we consider the case of Wallis ratio. We introduce some class of correction sequence $\left(\operatorname{MC}_{k}(n)\right)_{n \geq 1}$ such that the relative error sequence $E_{k}(n)$ has the fastest possible rate of convergence, which is defined by the relations

$$
\frac{(2 n-1) ! !}{(2 n) ! !}=\frac{1}{\sqrt{\pi\left(n+\frac{1}{4}\right)}} \exp \left(\operatorname{MC}_{k}(n)\right) \exp \left(E_{k}(n)\right), \quad k \in \mathbb{N}_{0}=\mathbb{N} \cup\{0\}
$$

The tool for measuring the rate of convergence is a result given by Mortici in [19], which says that a sequence $\left(x_{n}\right)_{n \geq 1}$ converging to zero is fastest possible when the difference $\left(x_{n}-\right.$ $\left.x_{n+1}\right)_{n \geq 1}$ is fastest possible. More precisely, if there exists $\lim _{n \rightarrow \infty} n^{\kappa}\left(x_{n}-x_{n+1}\right)=l$, with $\kappa>1$, then $\lim _{n \rightarrow \infty} n^{\kappa-1} x_{n}=\frac{l}{\kappa-1}$. Mortici's lemma has been effectively applied in many papers such as $[7,11,16,17,20-24]$. If $\lim _{n \rightarrow \infty} n^{\varrho} x_{n}=l \neq 0$ with constant $\varrho>0$, we say that the sequence $\left(x_{n}\right)_{n \geq 1}$ is order $n^{-\varrho}$, and write the exponent of convergence $\varrho=\varrho\left(x_{n}\right)$. Clearly if $\varrho\left(E_{k}(n)\right)=\varrho_{k}$, we have the following asymptotic formula:

$$
\frac{(2 n-1) ! !}{(2 n) ! !}=\frac{1}{\sqrt{\pi\left(n+\frac{1}{4}\right)}} \exp \left(\operatorname{MC}_{k}(n)\right)\left(1+O\left(n^{-\varrho_{k}}\right)\right)
$$

Let us briefly review the so-called multiple-correction method presented in our previous papers [16, 17]. Actually, the multiple-correction method is a recursive algorithm, and one of its advantages is that by repeating the correction process we always can accelerate the convergence, i.e., the sequence $\left(\varrho\left(E_{k}(n)\right)\right)_{k \geq 0}$ is strictly increasing. The key step is to find a suitable structure of $\mathrm{MC}_{k}(n)$. We often choose the $k$ th correction sequence $\mathrm{MC}_{k}(n)$ to be a finite generalized continued fraction (see [17] or (2.6) below) or a simple rational function (see [16] or (3.1) and (3.8) below).

It follows from (2.1) that

$$
\begin{aligned}
& E_{k}(n)=\ln W(n)+\frac{1}{2} \ln \pi+\frac{1}{2} \ln \left(n+\frac{1}{4}\right)-\mathrm{MC}_{k}(n), \\
& E_{k}(n)-E_{k}(n+1)=-\ln \frac{2 n+1}{2 n+2}-\frac{1}{2} \ln \frac{(n+1)+\frac{1}{4}}{n+\frac{1}{4}}+\mathrm{MC}_{k}(n+1)-\mathrm{MC}_{k}(n) .
\end{aligned}
$$

Next, we illustrate how to determine all the related coefficients in $\operatorname{MC}_{k}(n)$. The choice of the initial-correction sequence $\mathrm{MC}_{0}(n)$ is crucial. Since

$$
-\ln \frac{2 n+1}{2 n+2}-\frac{1}{2} \ln \frac{(n+1)+\frac{1}{4}}{n+\frac{1}{4}}=-\frac{1}{32 n^{3}}+O\left(\frac{1}{n^{4}}\right),
$$


in order to obtain the fastest possible sequence $E_{0}(n)-E_{0}(n+1)$, we choose $\mathrm{MC}_{0}(n)=$ $\frac{-\frac{1}{64}}{n^{2}+d_{1} n+d_{2}}$. Substituting this expression into (2.3) and expanding $E_{0}(n)-E_{0}(n+1)$ into a power series in terms of $n^{-1}$, by making use of Mathematica software we have

$$
\begin{aligned}
E_{0}(n)-E_{0}(n+1)= & \frac{\frac{3}{128}-\frac{3 d_{1}}{64}}{n^{4}}+\frac{-25+48 d_{1}+32 d_{1}^{2}-32 d_{2}}{512 n^{5}} \\
& -\frac{5\left(-31+64 d_{1}+64 d_{1}^{2}+32 d_{1}^{3}-64 d_{2}-64 d_{1} d_{2}\right)}{2,048 n^{6}}+O\left(\frac{1}{n^{7}}\right) .
\end{aligned}
$$

The fastest sequence $E_{0}(n)-E_{0}(n+1)$ is obtained by enforcing the first two coefficients of this power series to be zero. In this case, we find

$$
d_{1}=\frac{1}{2}, \quad d_{2}=\frac{7}{32}
$$

and hence

$$
E_{0}(n)-E_{0}(n+1)=-\frac{169}{32,768 n^{7}}+O\left(\frac{1}{n^{8}}\right)
$$

By Mortici's lemma, it is not difficult to verify that $\varrho\left(E_{0}(n)\right)=6$.

Just as in our previous works $[16,17]$, we can continue the above correction process to successively determine the correction sequence $\left(\mathrm{MC}_{k}(n)\right)_{n \geq 1}$ until some $k^{*}$ you want. On the one hand, to find the related coefficients avoiding huge computation, we often use appropriate symbolic computations software. On the other hand, the exact expressions at each occurrence also need a lot of space. Hence in this paper we omit many related details. Interested readers may refer to our previous papers $[16,17]$. Let

$$
\operatorname{MC}_{k}(n)=\underset{j=0}{k}\left(\frac{\kappa_{j}}{\left(n+\frac{1}{4}\right)^{2}+\lambda_{j}}\right), \quad k \in \mathbb{N}_{0}=\mathbb{N} \cup\{0\},
$$

where

$$
\begin{aligned}
& \kappa_{0}=-\frac{1}{64}, \quad \lambda_{0}=\frac{5}{32}, \\
& \kappa_{1}=-\frac{169}{3,072}, \quad \lambda_{1}=\frac{6,245}{5,408}, \\
& \kappa_{2}=-\frac{106,287,409}{109,674,240}, \quad \lambda_{2}=\frac{1,813,097,726,405}{574,802,307,872}, \\
& \kappa_{3}=-\frac{2,021,129,514,296,956,422,801}{404,884,957,099,688,791,040}, \\
& \lambda_{3}=\frac{27,813,569,451,306,412,085,060,531,045}{4,519,565,955,619,764,991,777,552,928} .
\end{aligned}
$$

By (2.3) and Mortici's lemma, we can use Mathematica software to check that $\varrho\left(E_{k}(n)\right)=$ $4 k+6$ for $0 \leq k \leq 3$. Thus,

$$
\frac{(2 n-1) ! !}{(2 n) ! !}=\frac{1}{\sqrt{\pi\left(n+\frac{1}{4}\right)}} \exp \left(\operatorname{MC}_{k}(n)\right)\left(1+O\left(n^{-4 k-6}\right)\right)
$$


Lastly, we remove the factor $\exp \left(E_{k}(n)\right)$ in (2.1) and further prove sharp double inequalities for as small $n$ as possible. Now we introduce another sequence $\operatorname{MD}_{k}(n)$ defined by

$$
\begin{aligned}
\operatorname{MD}_{0}(n) & =\frac{\kappa_{0}}{\left(n+\frac{1}{4}\right)^{2}}, \\
\operatorname{MD}_{k}(n) & =\frac{\kappa_{0}}{\left(n+\frac{1}{4}\right)^{2}+\lambda_{0}+} \cdots \frac{\kappa_{k-1}}{\left(n+\frac{1}{4}\right)^{2}+\lambda_{k-1}+} \frac{\kappa_{k}}{\left(n+\frac{1}{4}\right)^{2}} \quad \text { for } k \geq 1
\end{aligned}
$$

(i.e., $\operatorname{MD}_{k}(n)$ may be defined by taking the last coefficient $\lambda_{k}=0$ in the definition of $\left.\mathrm{MC}_{k}(n)\right)$, and improve inequalities (1.2) as follows.

Theorem 1 For $n \in \mathbb{N}$ and $k=0,1,2,3$, we have

$$
\frac{1}{\sqrt{\pi\left(n+\frac{1}{4}\right)}} \exp \left(\operatorname{MD}_{k}(n)\right) \leq \frac{(2 n-1) ! !}{(2 n) ! !}<\frac{1}{\sqrt{\pi\left(n+\frac{1}{4}\right)}} \exp \left(\operatorname{MC}_{k}(n)\right) .
$$

Proof We only give the proof in the case of $k=3$, other may be proved similarly. It suffices to show that $a(n)>0$ and $b(n)<0$, where

$$
a(n)=\ln W(n)+\frac{1}{2} \ln \pi+\frac{1}{2} \ln \left(n+\frac{1}{4}\right)-\mathrm{MD}_{k}(n)
$$

and

$$
b(n)=\ln W(n)+\frac{1}{2} \ln \pi+\frac{1}{2} \ln \left(n+\frac{1}{4}\right)-\operatorname{MC}_{k}(n) .
$$

As both $a(n)$ and $b(n)$ converge to zero, it suffices to prove that $(a(n))_{n \geq 1}$ is decreasing and $(b(n))_{n \geq 1}$ is increasing. We set $a(n+1)-a(n)=f(n)$ and $b(n+1)-b(n)=g(n)$, where

$$
f(x)=\ln \frac{2 x+1}{2 x+2}+\ln \frac{(x+1)+\frac{1}{4}}{x+\frac{1}{4}}-\mathrm{MD}_{k}(x+1)+\mathrm{MD}_{k}(x)
$$

and

$$
g(x)=\ln \frac{2 x+1}{2 x+2}+\ln \frac{(x+1)+\frac{1}{4}}{x+\frac{1}{4}}-\mathrm{MC}_{k}(x+1)+\mathrm{MC}_{k}(x) .
$$

By using Mathematica software, one may check

$$
f^{\prime}(x)=\frac{\Psi_{1}(17 ; x)(x-1)+14164 \ldots 23853}{\Psi_{2}(4 ; x)\left(\Psi_{3}(7 ; x)(x-1)+14752 \ldots 04469\right)^{2} \Psi_{4}(8 ; x)^{2}}>0 \quad(x \geq 1)
$$

and

$$
g^{\prime}(x)=-\frac{\Psi_{5}(16 ; x)^{2}}{(1+x)(1+2 x)(1+4 x)(5+4 x) \Psi_{6}(8 ; x)^{2} \Psi_{7}(8 ; x)^{2}}<0,
$$

where $14164 \ldots 23853$ and $14752 \ldots 04469$ denote two large positive constants. Thus, $f(x)$ is strictly increasing on $[1, \infty)$ with $f(\infty)=0$, while $g(x)$ is strictly decreasing on $[1, \infty)$ with $g(\infty)=0$. Hence $f<0$ and $g(x)>0$ for $x \geq 1$. This finishes the proof of Theorem 1 . 


\section{Inequalities for $T_{1}(n)$ and $T_{2}(n)$}

In this section, we will use the method of Section 2 to improve (1.5) and (1.6), respectively.

Theorem 2 Let $\beta$ be defined by (1.7). For $n \in \mathbb{N}$, we have

$$
\begin{aligned}
& \frac{\beta}{\sqrt[3]{n^{2}+\frac{1}{3} n+\frac{2}{27}}} \exp \left(\frac{\frac{4}{2,187}}{n^{4}+b_{1} n^{3}+b_{2} n^{2}+b_{3} n}\right) \\
& <\frac{1 \cdot 4 \cdots(3 n-2)}{3 \cdot 6 \cdots(3 n)}<\frac{\beta}{\sqrt[3]{n^{2}+\frac{1}{3} n+\frac{2}{27}}} \exp \left(\frac{\frac{4}{2,187}}{n^{4}+b_{1} n^{3}+b_{2} n^{2}+b_{3} n+b_{4}}\right)
\end{aligned}
$$

where

$$
b_{1}=\frac{2}{3}, \quad b_{2}=\frac{61}{81}, \quad b_{3}=\frac{52}{243}, \quad b_{4}=-\frac{1,742}{6,561} .
$$

Remark 1 It should be stressed that two kinds of correction processes are applied in (3.1). For comparison, we have

$$
\begin{aligned}
& T_{1}(n)=\frac{\beta}{\sqrt[3]{n^{2}+\frac{1}{3} n+\frac{2}{27}}}\left(1+O\left(\frac{1}{n^{4}}\right)\right), \\
& T_{1}(n)=\frac{\beta}{\sqrt[3]{n^{2}+\frac{1}{3} n+\frac{2}{27}}} \exp \left(\frac{\frac{4}{2,187}}{n^{4}+b_{1} n^{3}+b_{2} n^{2}+b_{3} n+b_{4}}\right)\left(1+O\left(\frac{1}{n^{10}}\right)\right) .
\end{aligned}
$$

Remark 2 We note that

$$
\begin{aligned}
& n^{2}+\frac{1}{3} n+\frac{2}{27}=\left(n+\frac{1}{6}\right)^{2}+\frac{5}{108} \\
& n^{4}+b_{1} n^{3}+b_{2} n^{2}+b_{3} n+b_{4}=\left(n+\frac{1}{6}\right)^{4}+\frac{95}{162}\left(n+\frac{1}{6}\right)^{2}-\frac{29,663}{104,976}
\end{aligned}
$$

Proof Since the proof of Theorem 2 is similar to that of Theorem 1, we only outline the idea of the proof here. Inequalities (3.1) are equivalent to

$$
\begin{aligned}
& u(n)=\ln T_{1}(n)-\ln \beta+\frac{1}{3} \ln \left(n^{2}+\frac{1}{3} n+\frac{2}{27}\right)-\frac{4 / 2,187}{n^{4}+b_{1} n^{3}+b_{2} n^{2}+b_{3} n}>0, \\
& v(n)=\ln T_{1}(n)-\ln \beta+\frac{1}{3} \ln \left(n^{2}+\frac{1}{3} n+\frac{2}{27}\right)-\frac{4 / 2,187}{n^{4}+b_{1} n^{3}+b_{2} n^{2}+b_{3} n+b_{4}}<0 .
\end{aligned}
$$

As both $u(n)$ and $v(n)$ converge to zero, it suffices to prove that $(u(n))_{n \geq 1}$ is decreasing and $(v(n))_{n \geq 1}$ is increasing. We write $u(n+1)-u(n)=\phi(n)$ and $v(n+1)-v(n)=\varphi(n)$, where

$$
\begin{aligned}
\phi(x)= & \ln \frac{3 x+1}{3 x+3}+\frac{1}{3} \ln \frac{(x+1)^{2}+\frac{1}{3}(x+1)+\frac{2}{27}}{x^{2}+\frac{1}{3} x+\frac{2}{27}} \\
& -\frac{4 / 2,187}{(x+1)^{4}+b_{1}(x+1)^{3}+b_{2}(x+1)^{2}+b_{3}(x+1)}+\frac{4 / 2,187}{x^{4}+b_{1} x^{3}+b_{2} x^{2}+b_{3} x}, \\
\varphi(x)= & \ln \frac{3 x+1}{3 x+3}+\frac{1}{3} \ln \frac{(x+1)^{2}+\frac{1}{3}(x+1)+\frac{2}{27}}{x^{2}+\frac{1}{3} x+\frac{2}{27}}
\end{aligned}
$$




$$
\begin{aligned}
& -\frac{4 / 2,187}{(x+1)^{4}+b_{1}(x+1)^{3}+b_{2}(x+1)^{2}+b_{3}(x+1)+b_{4}} \\
& +\frac{4 / 2,187}{x^{4}+b_{1} x^{3}+b_{2} x^{2}+b_{3} x+b_{4}} .
\end{aligned}
$$

By using Mathematica software again, we have for $x \geq 1$,

$$
\begin{aligned}
\phi^{\prime}(x) & =-\frac{64\left(\Psi_{1}(9 ; x)(x-1)+1,252,698,974,961,648,556\right)}{\Psi_{2}(6 ; x)\left(-1,742+1,404 x+4,941 x^{2}+4,374 x^{3}+6,561 x^{4}\right)^{2} \Psi_{3}(4 ; x)^{2}}>0, \\
\varphi^{\prime}(x) & =\frac{64\left(\Psi_{4}(9 ; x)(x-1)+1,514,366,592,000\right)}{9 x^{2}(1+x)^{2}(1+3 x)^{2}\left(2+9 x+27 x^{2}\right) \Psi_{5}(12 ; x)}<0 .
\end{aligned}
$$

Thus, $\phi(x)$ is strictly increasing on $[1, \infty)$ with $\phi(\infty)=0$, while $\varphi(x)$ is strictly decreasing on $[1, \infty)$ with $\varphi(\infty)=0$. Hence $\phi(x)<0$ and $\varphi(x)>0$ for $x \geq 1$. This completes the proof of Theorem 2.

Theorem 3 Let $\tau$ be defined by (1.7). For $n \in \mathbb{N}$, we have

$$
\frac{\tau}{\sqrt[3]{n+\frac{1}{3}}} \exp \left(-\frac{\frac{1}{81}}{n^{2}+\frac{2}{3} n}\right)<\frac{2 \cdot 5 \cdots(3 n-1)}{3 \cdot 6 \cdots(3 n)}<\frac{\tau}{\sqrt[3]{n+\frac{1}{3}}} \exp \left(-\frac{\frac{1}{81}}{\left(n+\frac{1}{3}\right)^{2}+\frac{1}{6}}\right) .
$$

Proof Similarly to the proof of Theorem 2, it suffices to show that for every integer $n \geq 1$,

$$
\begin{aligned}
& r(n)=\ln T_{2}(n)-\ln \tau+\frac{1}{3} \ln \left(n+\frac{1}{3}\right)+\frac{\frac{1}{81}}{n^{2}+\frac{2}{3} n}>0, \\
& s(n)=\ln T_{2}(n)-\ln \tau+\frac{1}{3} \ln \left(n+\frac{1}{3}\right)+\frac{\frac{1}{81}}{\left(n+\frac{1}{3}\right)^{2}+\frac{1}{6}}<0 .
\end{aligned}
$$

We let $r(n+1)-r(n)=\eta(n)$ and $s(n+1)-s(n)=\theta(n)$, where

$$
\begin{aligned}
& \eta(x)=\ln \frac{3 x+2}{3 x+3}+\frac{1}{3} \ln \frac{(x+1)+\frac{1}{3}}{x+\frac{1}{3}}+\frac{\frac{1}{81}}{(x+1)^{2}+\frac{2}{3}(x+1)}-\frac{\frac{1}{81}}{x^{2}+\frac{2}{3} x}, \\
& \theta(x)=\ln \frac{3 x+2}{3 x+3}+\frac{1}{3} \ln \frac{(x+1)+\frac{1}{3}}{x+\frac{1}{3}}+\frac{\frac{1}{81}}{\left(x+1+\frac{1}{6}\right)^{2}+\frac{1}{6}}-\frac{\frac{1}{81}}{\left(x+\frac{1}{6}\right)^{2}+\frac{1}{6}} .
\end{aligned}
$$

By using Mathematica software, we deduce that for every $x \geq 1$,

$$
\begin{aligned}
& \eta^{\prime}(x)=\frac{2\left(100+995 x+2,472 x^{2}+2,250 x^{3}+675 x^{4}\right)}{27 x^{2}(1+x)^{2}(1+3 x)(2+3 x)^{2}(4+3 x)(5+3 x)^{2}}>0, \\
& \theta^{\prime}(x)=-\frac{2\left(18,625+86,880 x+171,828 x^{2}+143,640 x^{3}+43,092 x^{4}\right)}{(1+x)(1+3 x)(2+3 x)(4+3 x)\left(5+12 x+18 x^{2}\right)^{2}\left(35+48 x+18 x^{2}\right)^{2}}<0 .
\end{aligned}
$$

We observe that both $r(n)$ and $s(n)$ converge to zero. Thus, $\eta(x)$ is strictly increasing on $[1, \infty)$ with $\eta(\infty)=0$, while $\theta(x)$ is strictly decreasing on $[1, \infty)$ with $\theta(\infty)=0$. Hence $\eta(x)<0$ and $\theta(x)>0$ for $x \geq 1$. This completes the proof of Theorem 3 .

Remark 3 In the proof of Theorems 2 and 3, we only use the initial-correction to improve (1.5) and (1.6). Certainly, we may further improve these inequalities by using multiplecorrection. 


\section{Continued fraction inequalities}

In this section, by changing the location of the correction sequence, we will employ the method of Section 2 to find the better continued fraction approximation. To do that, we introduce another correction sequence $\left(\operatorname{MC}_{k}\left(\frac{1}{2} ; n\right)\right)_{n \geq 1}$ and the relative error sequence $E_{k}\left(\frac{1}{2} ; n\right)$ with the following form:

$$
\frac{(2 n-1) ! !}{(2 n) ! !}=\frac{1}{\sqrt{\pi}} \frac{1}{\sqrt{n+\frac{1}{4}+\mathrm{MC}_{k}\left(\frac{1}{2} ; n\right)}} \exp \left(E_{k}\left(\frac{1}{2} ; n\right)\right) .
$$

Hence

$$
E_{k}\left(\frac{1}{2} ; n\right)-E_{k}\left(\frac{1}{2} ; n+1\right)=-\ln \frac{2 n+1}{2 n+2}-\frac{1}{2} \ln \frac{(n+1)+\frac{1}{4}+\mathrm{MC}_{k}\left(\frac{1}{2} ; n+1\right)}{n+\frac{1}{4}+\operatorname{MC}_{k}\left(\frac{1}{2} ; n\right)} .
$$

Let

$$
\operatorname{MC}_{k}\left(\frac{1}{2} ; n\right):={\underset{j}{j=0}}_{\mathbf{K}}^{k}\left(\frac{\kappa_{j}}{n+\lambda_{j}}\right), \quad k \in \mathbb{N}_{0}=\mathbb{N} \cup\{0\},
$$

where $\kappa_{0}=\frac{1}{32}, \kappa_{j}=\frac{(2 j+1)^{2}}{64}$ for $j \geq 1$, and $\lambda_{j}=\frac{1}{4}$ for $j \geq 0$. By applying the multiple-correction method described in Section 2, it is not difficult to check that if $0 \leq k \leq 20$, then

$$
\lim _{n \rightarrow \infty} n^{2 k+4} E_{k}\left(\frac{1}{2} ; n\right)=C_{k}\left(\frac{1}{2}\right)
$$

here $C_{k}\left(\frac{1}{2}\right)$ is a computable constant depending only on $k$.

Now we will state the general inequalities and their rates of convergence as follows.

Theorem 4 For $n \in \mathbb{N}$ and $k \in \mathbb{N} \cup\{0\}$, we have

$$
\frac{1}{\sqrt{\pi}} \frac{1}{\sqrt{n+\frac{1}{4}+\mathrm{MC}_{2 k}\left(\frac{1}{2} ; n\right)}}<\frac{(2 n-1) ! !}{(2 n) ! !}<\frac{1}{\sqrt{\pi}} \frac{1}{\sqrt{n+\frac{1}{4}+\mathrm{MC}_{2 k+1}\left(\frac{1}{2} ; n\right)}}
$$

and

$$
\varrho\left(E_{k}\left(\frac{1}{2} ; n\right)\right) \geq 2 k+4
$$

Proof By taking $x=4 n+1$ in Corollary 1 of Berndt [18], p.145, we obtain the so-called Lord Brouncker's continued fraction formula

$$
q(n):=\left(\frac{\Gamma\left(n+\frac{1}{2}\right)}{\Gamma(n+1)}\right)^{2}=\frac{4}{4 n+1+\frac{1^{2}}{2(4 n+1)+\frac{3^{2}}{2(4 n+1)+\frac{5^{2}}{2(4 n+1)+} \cdot}}} .
$$

After some simplification, we see that if $k \geq 2$, its $k$ th approximant $q_{k}(n)=\frac{A_{k}(n)}{B_{k}(n)}$ equals

$$
\left(\frac{1}{\sqrt{n+\frac{1}{4}+\mathrm{MC}_{k-2}\left(\frac{1}{2} ; n\right)}}\right)^{2} \text {. }
$$


As the partial coefficients of the continued fraction $q(n)$ are positive, we deduce inequalities (4.3) from (1.1) and (4.7) at once. Now, we are in a position to prove (4.4).

By the recurrence relations (1.10) we get

$$
q_{k+1}(n)-q_{k}(n)=\frac{A_{k+1}(n)}{B_{k+1}(n)}-\frac{A_{k}(n)}{B_{k}(n)}=\frac{(-1)^{k} 4 \prod_{j=1}^{k}(2 j-1)^{2}}{B_{k}(n) B_{k+1}(n)} .
$$

It follows from (4.8) and (4.7) that

$$
\left|q_{k}(n)-q(n)\right| \leq\left|q_{k+1}(n)-q_{k}(n)\right|=\frac{4 \prod_{j=1}^{k}(2 j-1)^{2}}{B_{k}(n) B_{k+1}(n)} .
$$

Also see equation (6) in [25]. The above use of alternating series technique is originated from Alf van der Poorten's lectures in [26]. Hence

$$
\left|\sqrt{q_{k}(n)}-\sqrt{q(n)}\right|<\frac{1}{\sqrt{q_{k}(n)}+\sqrt{q(n)}} \frac{4 \prod_{j=1}^{k}(2 j-1)^{2}}{B_{k}(n) B_{k+1}(n)},
$$

i.e.,

$$
\left|\frac{W(n)}{\sqrt{q_{k}(n)}}-1\right|<\frac{1}{\sqrt{q_{k}(n)}\left(\sqrt{q_{k}(n)}+W(n)\right)} \frac{4 \prod_{j=1}^{k}(2 j-1)^{2}}{B_{k}(n) B_{k+1}(n)} .
$$

We observe that $B_{k}(n)$ is a polynomial of degree $k$ in $n$, and $W(n), \sqrt{q_{k}(n)} \sim \frac{1}{2 \sqrt{n}}$. Now the assertion (4.4) follows from (1.1), (4.1), (4.8) and $\lim _{t \rightarrow 0} \frac{e^{t}-1}{t}=1$.

Theorem 4 tells us that Lord Brouncker's continued fraction formula also provides a better approximation for $W(n)$. It is a natural question whether there exists a similar continued fraction approximation formula for $\frac{\Gamma^{3}\left(x+\frac{1}{3}\right)}{\Gamma^{3}(x+1)}$ or $\frac{\Gamma^{3}\left(x+\frac{2}{3}\right)}{\Gamma^{3}(x+1)}$. To the best knowledge of authors, no one has even obtained such a kind of formulas, for example, see Berndt [18], Chapter 12. By the multiple-correction method and a large number of measurement data, we propose the following conjectures.

Open problems (i) There exist two sequences $\left(\kappa_{j}\right)_{j \geq 0}$ and $\left(\lambda_{j}\right)_{j \geq 0}$ of positive numbers such that

$$
\frac{\Gamma^{3}\left(x+\frac{1}{3}\right)}{\Gamma^{3}(x+1)}=\underset{j=0}{\mathbf{K}}\left(\frac{\kappa_{j}}{\left(x+\frac{1}{6}\right)^{2}+\lambda_{j}}\right), \quad x \geq 1,
$$

where

$$
\begin{aligned}
& \kappa_{0}=1, \quad\left(\kappa_{1}, \kappa_{2}, \kappa_{3}, \kappa_{4}, \ldots\right)=-\frac{2}{3^{6}}\left(1, \frac{2^{3} 5^{3}}{3^{2}}, \frac{4^{3} 7^{3}}{5^{2}}, \frac{5^{3} 11^{3}}{7^{2}}, \frac{7^{3} 13^{3}}{9^{2}}, \ldots\right), \\
& \left(\lambda_{0}, \lambda_{1}, \lambda_{2}, \lambda_{3}, \lambda_{4}, \ldots\right)=\frac{1}{2^{2} 3^{3}}\left(5, \frac{5^{2}}{1 \cdot 3}, \frac{3,307}{3 \cdot 5}, \frac{17,167}{5 \cdot 7}, \frac{5 \cdot 31 \cdot 353}{7 \cdot 9}, \ldots\right) .
\end{aligned}
$$

(ii) We have

$$
\frac{\Gamma^{3}\left(x+\frac{2}{3}\right)}{\Gamma^{3}(x+1)}=\underset{j=0}{\mathbf{K}}\left(\frac{v_{j}}{x+\frac{1}{3}}\right), \quad x \geq 1,
$$


where $v_{0}=1, v_{1}=\frac{1}{27}$, and for $j \geq 2$,

$$
\begin{aligned}
& v_{j}=\frac{1}{54} \frac{\left(j+\left\lfloor\frac{j-1}{2}\right\rfloor\right)^{3}}{2\left\lfloor\frac{j-1}{2}\right\rfloor+1}, \\
& \quad \text { i.e. }\left(v_{2}, v_{3}, v_{4}, \ldots\right)=\frac{1}{54}\left(\frac{2^{3}}{1}, \frac{4^{3}}{3}, \frac{5^{3}}{3}, \frac{7^{3}}{5}, \frac{8^{3}}{5}, \frac{10^{3}}{7}, \frac{11^{3}}{7}, \frac{13^{3}}{9}, \frac{14^{3}}{9}, \frac{16^{3}}{11}, \ldots\right) .
\end{aligned}
$$

Remark 4 It seems difficult to prove the above conjectures. However, by the same method as the proof of Theorem 1 , we can prove many sharp inequalities, e.g., for $n \in \mathbb{N}$ and $0 \leq$ $k \leq 10$, we have

$$
\frac{\tau}{\sqrt[3]{n+\frac{1}{3}+\mathbf{K}_{j=1}^{2 k+1}\left(\frac{v_{j}}{n+\frac{1}{3}}\right)}}<T_{2}(n)<\frac{\tau}{\sqrt[3]{n+\frac{1}{3}+\mathbf{K}_{j=1}^{2 k}\left(\frac{v_{j}}{n+\frac{1}{3}}\right)}}
$$

Competing interests

The authors declare that they have no competing interests.

Authors' contributions

All authors read and approved the final manuscript.

\section{Acknowledgements}

The authors would like to thank the referees for their careful reading of the manuscript and insightful comments which led to improvements of our original manuscript. The research of this paper was supported by the National Natural Science Foundation of China (Grant No. 11171344) and the Natural Science Foundation of Beijing (Grant No. 1112010).

Received: 2 March 2015 Accepted: 19 May 2015 Published online: 03 June 2015

\section{References}

1. Chen, C-P, Qi, F: The best bounds in Wallis' inequality. Proc. Am. Math. Soc. 133, 397-401 (2005)

2. Guo, S, Xu, J, Qi, F: Some exact constants for the approximation of the quantity in the Wallis' formula. J. Inequal. Appl. 2013, 67 (2013)

3. Guo, B-N, Qi, F, Zhao, J-L, Luo, Q-M: Sharp inequalities for polygamma functions. Math. Slovaca 65(1), 103-120 (2015). doi:10.1515/ms-2015-0010

4. Lampret, $\mathrm{V}$ : Wallis sequence estimated through the Euler-Maclaurin formula: even from the Wallis product $\pi$ could be computed fairly accurately. Aust. Math. Soc. Gaz. 31, 328-339 (2004)

5. Lampret, V: An asymptotic approximation of Wallis' sequence. Cent. Eur. J. Math. 10, 775-787 (2012)

6. Luke, YL: The Special Functions and Their Approximations, vol. I. Academic Press, New York (1969)

7. Mortici, C, Cristea, VG, Lu, D: Completely monotonic functions and inequalities associated some ratio of gamma function. Appl. Math. Comput. 240, 168-174 (2014)

8. Mortici, C: New approximation formulas for evaluating the ratio of gamma functions. Math. Comput. Model. 52 , 425-433 (2010)

9. Qi, F: Bounds for the ratio of two gamma functions. J. Inequal. Appl. 2010, Article ID 493058 (2010)

10. Qi, F, Luo, Q-M: Bounds for the ratio of two gamma functions: from Wendel's asymptotic relation to Elezović-Giordano-Pečarić's theorem. J. Inequal. Appl. 2013, 542 (2013)

11. Qi, F, Mortici, C: Some best approximation formulas and inequalities for the Wallis ratio. Appl. Math. Comput. 253 , 363-368 (2015). doi:10.1016/j.amc.2014.12.039

12. Qi, F: Bounds for the ratio of two gamma functions: from Gautschi's and Kershaw's inequalities to complete monotonicity. Turk. J. Anal. Number Theory 2(5), 152-164 (2014). doi:10.12691/tjant-2-5-1

13. Qi, F, Luo, Q-M: Complete monotonicity of a function involving the gamma function and applications. Period. Math. Hung. 69(2), 159-169 (2014). doi:10.1007/s10998-014-0056-x

14. Abramowitz, M, Stegun, IA (eds.): Handbook of Mathematical Functions with Formulas, Graphs, and Mathematical Tables, 9th edn. Applied Mathematics Series, vol. 55. National Bureau of Standards, Washington (1972)

15. Chen, C-P, Qi, F: Completely monotonic functions associated with the gamma functions and proof of Wallis' inequality. Tamkang J. Math. 36(4), 303-307 (2005)

16. Cao, XD, Xu, HM, You, X: Multiple-correction and faster approximation. J. Number Theory 149, $327-350$ (2015). doi:10.1016/j.jnt.2014.10.016

17. Cao, XD: Multiple-correction and continued fraction approximation. J. Math. Anal. Appl. 424, 1425-1446 (2015). doi:10.1016/j.jmaa.2014.12.014

18. Berndt, BC: Ramanujan's Notebooks - Part II. Springer, Berlin (1989)

19. Mortici, C: Product approximations via asymptotic integration. Am. Math. Mon. 117(5), 434-441 (2010)

20. Mortici, C, Chen, C-P: New sequence converging towards the Euler-Mascheroni constant. Comput. Math. Appl. 64(4), 391-398 (2012)

21. Mortici, C: Ramanujan formula for the generalized Stirling approximation. Appl. Math. Comput. 217(6), $2579-2585$ (2010) 
22. Mortici, C: On Gospers formula for the gamma function. J. Math. Inequal. 5(4), 611-614 (2011)

23. Mortici, C: On Ramanujan's large argument formula for the gamma function. Ramanujan J. 26(2), 185-192 (2011)

24. Mortici, C: Improved asymptotic formulas for the gamma function. Comput. Math. Appl. 61(11), 3364-3369 (2011)

25. Hančl, J, Leppälä, K, Matala-aho, T, Törmä, T: On irrationality exponents of generalized continued fractions. J. Number Theory 151, 18-35 (2015). doi:10.1016/j.jnt.2014.09.034

26. van der Poorten, AJ: An introduction to continued fractions. In: Diophantine Analysis (Kensington, 1985). London Math. Soc. Lecture Note Ser., vol. 109, pp. 99-138. Cambridge University Press, Cambridge (1986)

Submit your manuscript to a SpringerOpen ${ }^{\odot}$ journal and benefit from:

- Convenient online submission

- Rigorous peer review

- Immediate publication on acceptance

- Open access: articles freely available online

- High visibility within the field

- Retaining the copyright to your article

Submit your next manuscript at springeropen.com 